\title{
Pas d'angoisse de la feuille blanche
}

\section{Kai Felmy}

Dessinateur

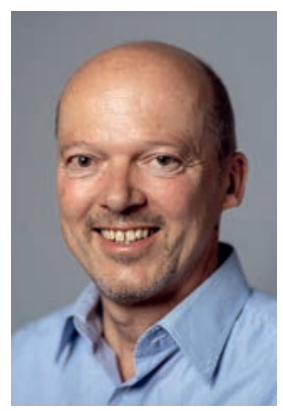

Bonjour chers lecteurs, je m'appelle Kai Felmy. Je suis né le 8 juillet 1963 à Francfort-sur-le-Main. Mon intérêt pour le dessin s'est développé très tôt et, dès mes sept ans, je créais mes premiers personnages à gros pif, les «Knollies». Ils ne se sont toutefois imposés que trente ans plus tard.

J'ai eu la chance de pouvoir faire de mon don de boute-en train dès l'école primaire (surtout auprès des professeurs de math quand j'essayais de résoudre un problème) mon métier. Mais j'aurais aussi pu devenir musicien. A 14 ans, je me produisais dans des bars avec mon groupe de rock.

Après ma formation de graphiste, j'ai travaillé dans plusieurs agences de publicité de Francfort, d'abord en tant qu'illustrateur, puis en tant que directeur artistique. Mon premier dessin est paru en 1988 dans le cadre d'une campagne publicitaire de la Frankfurter Allgemeine Zeitung (FAZ).

En 1995, je me suis lancé en tant que dessinateur indépendant. Depuis 1999, plus de 3000 de mes dessins ont été publiés dans la rubrique économique suprarégionale de la FAZ. Des reproductions dans de multiples revues, journaux et livres en Allemagne, en Autriche et en Suisse, puis plus tard des publications dans des médias en ligne ont suivi. A lui seul, le supplément TV rtv, pour lequel j'ai croqué une illustration hebdomadaire pendant une dizaine d'années, était initialement tiré à neuf millions d'exemplaires. Si seulement j'avais négocié des honoraires d'un euro par exemplaire... J'ai également publié dix livres au total, il y a maintenant plusieurs années de cela.

Comme chacun sait, tout commence par une page blanche! Lorsqu'on veut vivre du métier d'illustrateur, il faut recevoir beaucoup de commandes, dessiner de nombreux cartoons et proposer encore davantage d'idées. Le tout de préférence le plus vite possible. Travailler pour les quotidiens, dont certains me confiaient il y a quelques années souvent deux, voire parfois trois (!!!) commandes par jour en lien avec des articles précis, a été une expérience particulièrement éprouvante (mais aussi gratifiante et enrichissante). Il fallait donc lire trois textes, développer cinq à sept ébauches d'idées par commande, les faxer (ce qui allait plus vite que de les scanner et les envoyer par mail), finaliser la sélection des rédacteurs - le plus souvent en noir et blancet la leur faxer (toujours pour gagner du temps). Tout cela entre $9 \mathrm{~h}$ du matin et le bouclage de la rédaction, à
Les trois dessins paraissaient le jour suivant dans le journal. Au niveau suprarégional et avec un tirage de 350000 à 450000 exemplaires par jour. Ça vous maintient jeune! On a l'impression d'être passé en alternance sous l'eau chaude et froide pendant des heures. D'avoir été complètement lessivé, essoré, défroissé et miraculeusement ramené à la vie. Après cela, on n'a plus l'angoisse de la page blanche (parce qu'on n'a tout simplement pas le temps d'y penser).

Mes dessins ont surtout pour but de faire rire, mais parfois aussi d'exposer les choses sous un nouveau jour et/ ou de présenter un point de vue différent et inhabituel. A mon sens, une illustration est bonne lorsqu'elle repose sur une idée surprenante et drôle. Un dessin amusant ne suffit pas à faire un bon cartoon.

Pour moi, l'image et le texte doivent dans l'idéal se compléter. Il ne s'agit donc pas de redire avec des mots ce qu'exprime déjà le dessin, mais de donner au lecteur de l'espace pour associer image et texte. Il se produit alors un déclic et, dans le meilleur des cas, on en rit. Surtout lorsqu'on force le trait d'une scène caractéristique connue, ou qu'on la présente de manière décalée. Mes inspirations sont Uli Stein, Loriot et Gary Larson. Ce dernier est capable de raconter des histoires entières en une seule image. Chaque année à la Saint-Sylvestre, je tombe sur Dinner for one (avec Freddie Frinton et May Warden) et je m'écroule de rire sous la table.

Quand est-ce que je cesse de rire? Quand je regarde mon relevé bancaire à la fin du mois (ha ha... je plaisante).

Aujourd'hui, je m'en sors bien avec une à deux commandes par jour (les quotidiens sont devenus l'exception), j'ai même le temps de boire un café entre deux, de faire des exercices pour le dos (parfaits quand on passe beaucoup de temps assis) et de faire du sport le soir. Je suis heureux dans ma vie de divorcé, plus heureux encore d'avoir retrouvé quelqu'un, j'ai trois enfants adultes et deux chiens et j'habite une petite maison à la campagne, près de Francfort (là où il n'y a pas encore trop de feux de circulation).

Mon lien avec la médecine? Je pense que c'est un sujet qui nous concerne tous. Tout le monde a très probablement déjà été malade. Et c'est un domaine où un peu d'humour ne fait pas de mal. Ne dit-on pas que le rire, c'est la santé? Peut-être mes dessins peuvent-ils contribuer à guérir ou à égayer le quotidien des professionnels de la santé. Cela donnerait un très beau sens à mon travail. Dans cet esprit, je vous souhaite de bien rire devant mes illustrations et de rester en bonne santé! 